\title{
Relation between Interleukin 8 and Bronchial Asthma in Children: Review Article
} Sahbaa Fehr Mohamed ${ }^{1}$, Fathy Mohamed Abd-Elwahab*1, Dina Mohamed Shokry ${ }^{1}$, Walaa Mohamed-Samy ${ }^{2}$ Departments ${ }^{1}$ Pediatrics and ${ }^{2}$ Medical Biochemistry, Faculty of Medicine, Zagazig University, Egypt.

*Corresponding Author: Fathy Mohamed Abd-elwahab, Email: drfathy2007@ gmail.com

\begin{abstract}
Background: Asthma is a frequent respiratory condition to treat. A persistent airway inflammation characterizes this frequent form of pulmonary disease. Immune responses are triggered by cytokines and chemokines produced by airway epithelial cells. Human bronchial epithelial cells secrete IL-8 in response to the presence of interleukin-4 (IL-4) and interleukin-13, both of which are increased in asthmatics. There are two receptors for IL-8, the IL-8 receptor alpha (also known as CXCR1) and beta (also known as the IL-8 RB, CXCR2). IL8 is a potent chemotactic cytokine that activates inflammatory cells by recruiting mast cells, mononuclear phagocytes $\mathrm{T}$ lymphocytes, and neutrophils to the site of inflammation. Objective: To determine the relationship between IL8 and bronchial asthma in children.

Conclusion: The assessment of IL8 levels in pediatric asthmatic patients is a useful biomarker reflecting the status of asthma and also to glucocorticoids and treatment responses.
\end{abstract}

Keywords: Bronchial asthma, Interleukin 8.

\section{Bronchial asthma in pediatrics:}

Asthma is a diverse disease that is often accompanied with persistent inflammation of the respiratory tracts. Chest tightness, shortness of breath and cough, and wheezes, which change in time and intensity over time, and variable expiratory airflow limitation are used to identify the disease ${ }^{(\mathbf{1})}$.

\section{Pathogenesis of asthma:}

Individuals with allergic asthma, who are predisposed genetically, respond to allergens by mounting an incorrect T-cell immune response, resulting in chronic airway narrowing. Airway hyper-responsiveness to a wide range of particular and non-specific stimuli characterizes allergic asthma, which is defined by the formation of inflammatory infiltrates in the lungs and airway hyper-responsiveness to a variety of specific and nonspecific stimuli, a rise in Immunoglobulin E (IgE) levels in the bloodstream, and increased mucus production. As a result of the chronic inflammation, structural changes (airway remodeling) occur, including collagen deposition, hyperplasia, and thickening of the airway wall. The bronchoalveolar infiltration of numerous immune cell types, predominantly eosinophils, mast cells and activated $\mathrm{CD}^{+}{ }^{+} \mathrm{T}$-cells, is triggered in asthmatic patients by allergic episodes. To coordinate the immune response to allergens, effector Thelper 2 (Th2) cells release cytokines that set off the hallmarks of asthma. There are several factors that contribute to the formation of $\mathrm{IgE}$, including the secretion of interleukin 4 and interleukin 13 , as well as the activation of mast cells by interleukin 5 and $9^{(2)}$.

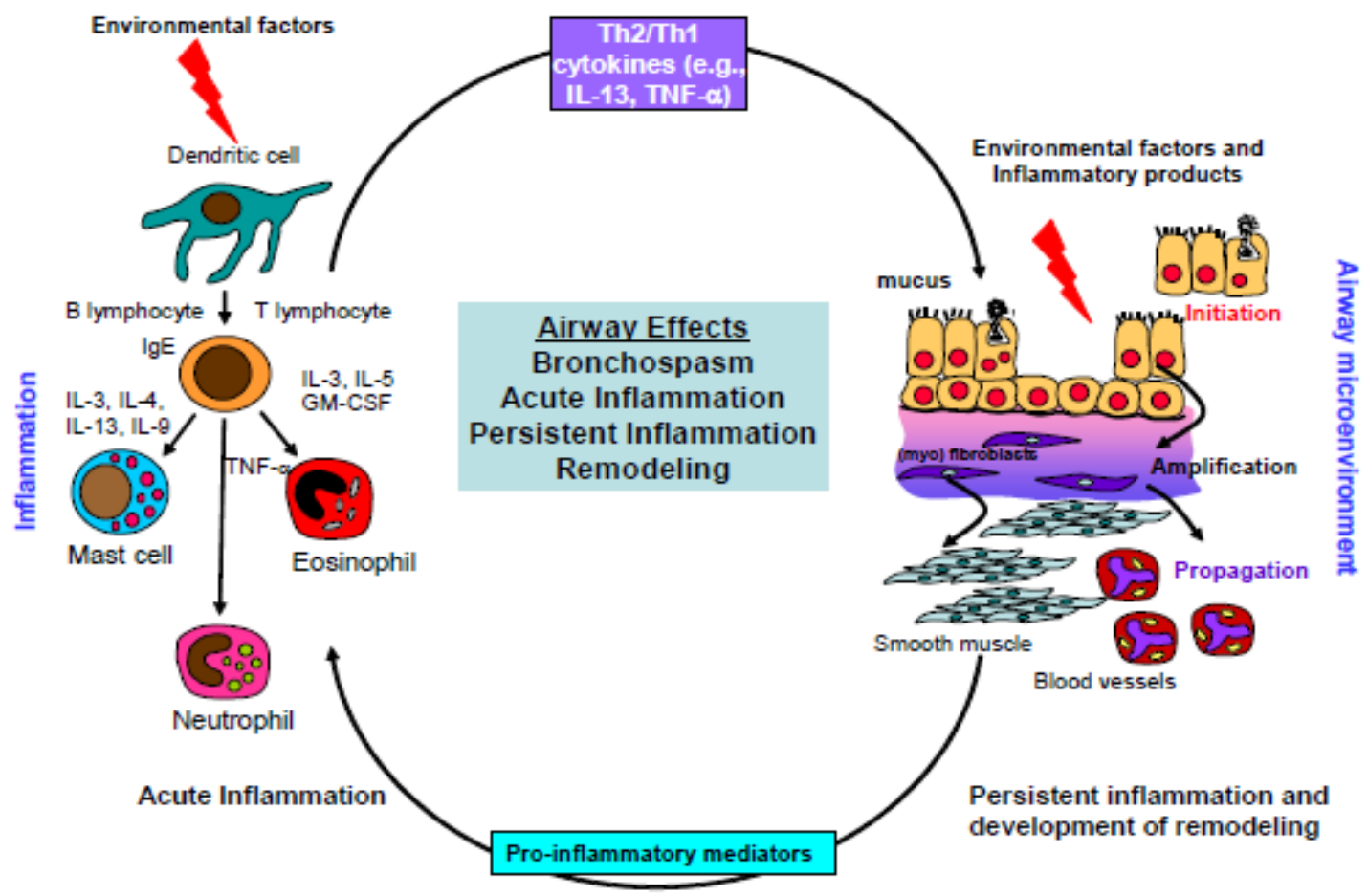

Figure (1): Factors limiting airflow in acute and persistent asthma ${ }^{(2)}$.

This article is an open access article distributed under the terms and conditions of the Creative Commons Attribution (CC BY-SA) license (http://creativecommons.org/licenses/by/4.0/) 
When IL-4 and IL-13 act on lung epithelial cells, they promote goblet cell metaplasia while IL-13 acts on smooth muscle cells, it helps to establish airway hyperresponsiveness in the body. Other T-helper cell subsets, such as Th9, Th25, and Th22 cells, have been connected to the etiology of asthma ${ }^{(3)}$.

Th17 cells, a subset of lung-infiltration T-cells, have been identified as being responsible for neutrophilic airway inflammation as well as increased Th2-cell induced eosinophilic airway inflammation. Asthma patients' Th17 cells and eosinophils secrete more IL-17, which was also found to be upregulated ${ }^{(4)}$.

The innate immune system also contributes to the pathophysiology of asthma by producing proinflammatory mediators via bronchial epithelial cells, mast cells, basophils, and Natural Killer T (NKT) and dendritic cells, but asthma is often considered an adaptive immunological condition ${ }^{(5)}$.

There's plenty of evidence to suggest that suppressive and regulatory systems play a critical role in keeping healthy people's lungs free of detrimental responses ${ }^{(6)}$.

\section{Interleukin 8 and asthma:}

Neutrophil chemotactic factor, or IL-8, is a protein that has two purposes. Target cells, mostly neutrophils but also other granulocytes, are induced to undergo chemotaxis, prompting them to go toward the infection site. Once they arrive, IL-8 triggers phagocytosis. Il-8 is also well-known for its ability to stimulate angiogenesis. IL-8 activates a number of physiological reactions in target cells, including increases in intracellular $\mathrm{Ca}^{2+}$ and exocytosis, which are both necessary for cell motility and phagocytosis ${ }^{(7)}$.

It is thought that IL-8 has a role in the pathogenesis of bronchiolitis, a virally induced illness of the upper respiratory tract. CXC chemokines, including IL-8, are found throughout the body. The CXC chemokine genes, along with the genes for the other eight members of the family, are located in a cluster on chromosome $4 q^{(7)}$.

There have been several inflammatory disorders linked to IL-8, including asthma, ARDS (adult respiratory distress syndrome), cystic fibrosis (CF), COPD (chronic obstructive pulmonary disease). The airway epithelium is a major producer of the antimicrobial cytokine IL-8, and it protects the airway from microorganism invasion. By encouraging neutrophil chemotaxis and inflammatory responses in the airways, IL- 8 released by the airway epithelium helps the host body fight infection and disease ${ }^{(\mathbf{8})}$.

While infancy is a critical period for the development of asthma and wheezing, it may also lead to recurrent wheeze and/or bronchial asthma due to the function that IL-8 plays in these conditions ${ }^{(9)}$.

Bronchoalveolar lavage (BAL) fluid and sputum from asthmatic patients have higher levels of IL-8. Bronchial hyperreactivity in guinea pigs is caused by repeated inhalation of IL-8. Researchers have discovered a link between asthma and IL- 8 on the genome ${ }^{(\mathbf{1 0})}$.

There are two kinds of receptors for IL-8: IL-8 alfa receptor (IL-8RA, CXCR1) and (IL-8RA, CXCR2) (IL-8RB, CXCR2). Receptors and guanine nucleotidebinding proteins belong to the same superfamily and have a close relationship. The IL-8RA gene resides on chromosome $2 \mathrm{q} 35$, where it has been linked to higher levels of total serum IgE in asthmatic patients ${ }^{(11)}$.

A study conducted by Nocker $\boldsymbol{e t}$ al. ${ }^{(12)}$ examined if IL-8 was present in the secretions of asthmatic patients to learn more about the disease's connection to airway inflammation. Asthma patients with clinical stability had higher amounts of IL-8 in their bronchoalveolar lavage fluid (BALF) compared to healthy controls. Patients with asthma had higher IL-8 levels when their BALF neutrophil count was higher. The higher concentrations of the inflammatory mediator interleukin-8 in asthmatic patients' secretions could be signs of a continuous inflammation. The substantial link between IL-8 levels and neutrophil percentages and/or myeloperoxidase (MPO) levels in asthma patients suggests that IL-8 plays a role in the recruitment and activation of neutrophils in the airway lumen in asthma.

According to Wang et al. ${ }^{\left({ }^{(3)}\right)}$, patients with COPD had significantly higher levels of sputum IL-8 than healthy controls. These patients' forced expiratory volume in the first second (FEV1) and sputum IL-8 levels were shown to be negatively correlated. Asthmatic patients had higher levels of serum and BAL IL-8 than normal participants, according to Stankiewicz et al. ${ }^{(14)}$. According to Govindaraju et al. (15), asthmatic patients had significantly higher levels of IL-8 in their plasma and sputum than healthy controls. Patients with COPD have higher levels of IL-8 in their airway secretions, which could be a sign of a continuous inflammatory process that is more severe in those with COPD. Neutrophils in asthma are activated by IL-8, which can be used as a marker for the degree of airway inflammation.

Before and after prednisolone medication, Tang and Chen (16) found variations in IL-8 blood concentrations created by activated eosinophils. With the help of commercially available test kits, they measured the concentration of IL-8 in the blood of 15 healthy control participants and in sera from 20 allergic asthmatic children who were experiencing an acute exacerbation but were otherwise healthy. Asthmatic children with acute exacerbations and stable asthmatics had considerably higher mean serum IL-8 concentrations than participants in the control group. However, there was no statistically significant difference between the groups of those with acute exacerbations and those who had stable asthma. Because serum IL-8 content is a poor predictor of disease activity in acute asthma, measuring it has limited usefulness.

The role of interleukin-8 (IL-8) in the etiology of bronchial asthma was studied by Alwakil et al. ${ }^{(17)}$. This study found that the interleukin-6 (IL-6) was implicated 
in the development of asthma. This cytokine is involved in asthmatic patients' inflammatory alterations. As a result, bronchial asthma sufferers may experience a worsening of their symptoms if they are exposed to IL8.

Using blood interleukin-8 (IL-8) levels as a biomarker for uncontrolled asthma, Zhang and Bai ${ }^{(18)}$ analysed. They discovered that a rise in serum IL-8 levels is a good biomarker for determining whether or not an asthmatic is receiving therapy for the condition. The change in IL-8 level in uncontrolled asthma also reflects the response to glucocorticoids. Inflammation may be linked to asthma pathogenesis, according to these preliminary findings.

\section{CONCLUSION}

The assessment of IL8 levels in pediatric asthmatic patients is a useful biomarker reflecting the status of asthma and also to glucocorticoids and treatment responses.

Financial support and sponsorship: Nil.

Conflict of interest: Nil.

\section{REFERENCES}

1. Global Initiative for Asthma (2017): Global strategy for asthma management and prevention in children. Based on the Workshop Report. https://www.who.int/respiratory/asthma/GINA_WR_2 006_copyright $\% 5 B 1 \% 5 \mathrm{D} . p d f$

2. Rosenberg H, Phipps S, Foster P (2007): Eosinophil trafficking in allergy and asthma. J. Allergy Clin. Immunol., 119: 1303-1312.

3. Nakagome $\mathrm{K}$, Imamura $\mathrm{M}$, Kawahata $\mathrm{K}$ et al. (2011): High expression of IL-22 suppresses antigeninduced immune responses and eosinophilic airway inflammation via an IL-10-associated mechanism. J. Immunol., 187: 4077-5089.

4. Levine E, Wenzel E (2010): The role of Th2 immune pathway modulation in the treatment of severe asthma and its phenotypes. Ann Intern Med., 152(4): 232-237.

5. Ono E, Tanguchi M, Higashi $\mathbf{N}$ et al. (2010): CD23 expression on human basophils is associated with asthma exacerbation. J. Alelrgy Clin Immunol., 125: 483-489.

6. Ray A, Khare A, Krishnamoorthy $\mathrm{N}$ et al. (2010): Regulatory $\mathrm{T}$ cells in many flavors control asthma. Afucosal Immunol., 3: 216-229.
7. Brat D, Bellail A, Van Meir E (2005): The role of interleukin-8 and its receptors in gliomagenesis and tumoral angiogenesis. Neuro-oncol., 7: 122-33.

8. Donnelly S, Strieter R, Kunkel S et al. (1993): Interleukin-8 and development of adult respiratory distress syndrome in at-risk patient groups. Lancet, 341(8846): 643-647.

9. Sigurs N, Gustafsson P, Bjarnason $R$ et al. (2005): Severe respiratory syncytial virus bronchiolitis in infancy and asthma and allergy at age 13. American Journal of Respiratory and Critical Care Medicine, 171(2): 137-141.

10. Heinzmann A, Ahlert I, Kurz T et al. (2004): Association study suggests opposite effects of polymorphisms within IL8 on bronchial asthma and respiratory syncytial virus bronchiolitis. J Allergy Clin Immunol., 114: 671-676.

11. Hemant S, Sharma $H$ (2008): A comparison of skin prick tests, intradermal skin tests, and specific IgE in the diagnosis of mouse allergy. American Academy of Allergy, Asthma and Immunology, 10: 933-939.

12. Nocker R, Schoonbrood D, van de Graaf E et al. (1996): Interleukin-8 in airway inflammation in patients with asthma and chronic obstructive pulmonary disease. Int Arch Allergy Immunol., 109(2): 183-91.

13. Wang S, Xu F, Chen Y (2000): Detection and significance of interleukin-8, 6 , tumor necrosis factor- $\alpha$ in sputa from patients with chronic obstructive pulmonary disease. Zhonghua Jie He He Hu Xi Za Zhi., 23(8):472-474.

14. Stankiewicz W, Dabrowski M, Chcialowski A et al. (2002): Cellular and cytokine immunoregulation in patients with chronic obstructive pulmonary disease and bronchial asthma. Mediators Inflamm., 11(5):307-312.

15. Govindaraju V, Michoud $M$, Al Chalabi $M$ et al. (2006): Interleukin-8: novel roles in human airway smooth muscle cell contraction and migration. Am J Physiol Cell Physiol., 291: 957-965.

16. Tang R, Chen S (2009): Evaluation of serum interleukin-8 as a marker of disease activity in acute asthma in children. Journal of Asthma, 9: 409-413.

17. Alwakil I, Al Kabeer A, Kabil T et al. (2011): Serum interleukin-6 and interleukin-8 in bronchial asthma. AAMJ., 9(3): 385-396.

18. Zhang J, Bai C (2017): Elevated serum interleukin-8 level as a preferable biomarker for identifying uncontrolled asthma and glucocorticosteroid responsiveness. Tranaffos., 15(4): 260-269. 